\title{
Exposure assessment in different occupational groups at a hospital using Quick Exposure Check (QEC) - A pilot study
}

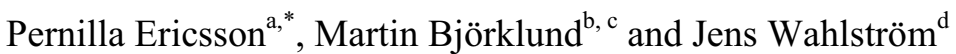 \\ ${ }^{\mathrm{a}}$ Work and Environmental Medicine, Umeå University Hospital, SE-901 85 Umeå, Sweden, ${ }^{\mathrm{b}}$ Centre for \\ Musculoskeletal Research, Department of Occupational and Public Health Sciences, University of Gävle, SE-801 \\ 76 Gävle, Sweden, ${ }^{\mathrm{c}}$ Department of Community Medicine and Rehabilitation, Physiotherapy, Umeå University, \\ SE-901 87 Umeå, Sweden, ${ }^{\mathrm{d}}$ Department of Public Health and Clinical Medicine, Occupational and \\ Environmental Medicine, Umeå University, SE-901 85 Umeå, Sweden
}

\begin{abstract}
In order to test the feasibility and sensitivity of the ergonomic exposure assessment tool Quick Exposure Check (QEC), a pilot-study was conducted. The aim was to test QEC in different occupational groups to compare the exposure in the most common work task with the exposure in the work task perceived as the most strenuous for the neck/shoulder region, and to test intra-observer reliability. One experienced ergonomist observed 23 workers. The mean observation time was 45 minutes, waiting time and time for complementary questions included. The exposure scores varied between the different occupational groups as well as between workers within the occupational groups. Eighteen workers rated their most common work task as also being the most strenuous for the neck/shoulder region. For the remaining five workers, the mean exposure score were higher both for the neck and shoulder/arm in the most common work task. Intra-observer reliability shows agreement in $86 \%$ of the exposure interactions in the neck and in $71 \%$ in the shoulder/arm. QEC seems to fulfill the expectations of being a quick, sensible and practical exposure assessment tool that covers physical risk factors in the neck, upper extremities and low back.
\end{abstract}

Keywords: exposure assessment, risk assessment, observations

\section{Introduction}

Biomechanical risk factors such as excessive repetition, heavy lifting and awkward postures may cause work-related musculoskeletal disorders [1,2]. One of the most used methods to identify hazards at work and evaluating the effect of ergonomic changes are observational methods [3]. Physical exposure should be assessed in three dimensions; intensity, frequency and duration [4, 5]. Ergonomic work-place interventions should implicate known biomechanical and psychosocial risk factors, and include experts and workers assessed [2].
The Quick Exposure Check (QEC) is based on scientific evidence, and has been developed the past 15 years with the goal to be a user friendly ergonomic risk assessment tool usable for practitioners [6, 7]. QEC has been found to have good validity and moderate inter- and intra-observer reliability $[3,7]$

We are planning to use QEC to assess ergonomic exposure in a randomized controlled trial studying effects of individualized treatment on neck pain. In order to test the feasibility and sensitivity of QEC, a pilot-study was conducted. The aim was to test QEC

*Corresponding author. E-mail: pernilla.eriksson@vll.se 
in different occupational groups at a hospital in Sweden, to compare the exposure in the most common task with the exposure in the work task perceived as the most strenuous for the neck/shoulder region and to test intra-observer reliability.

\section{Methods}

In the study 23 workers ( 22 women), mean age 47 (range 25-62), volunteered to participate. In total, five operating-room nurses, five medical secretaries, five workers at the hospital equipment sterilization department, four hospital cleaners and four biomedical analysts (BMA) participated.

The workers assessed their most common work task, the most strenuous work task for the neck/shoulder region, and the time spent on each work task in an ordinary work day. They also answered complementary questions regarding their physical and psychosocial work environment.

Direct observations were performed in all 23 workers by an experienced ergonomist (PE, first author) when the workers carried out the different work tasks. Fourteen of the workers volunteered to be videotaped during their work in order to make reassessments.

Observations were performed regarding risk factors of the neck (posture), back (posture and frequency of movement), shoulder/arm (posture and frequency of movement), and wrist/hand (posture and frequency of movement). The workers rated risk factors such as load weight, duration, force and visual demands subjectively. The total exposure score in each body region was calculated as the sum of 2-5 interactions for example posture $x$ duration and frequency $x$ duration. Ranges of possible scores were for the neck 4-18 and shoulder/arm 10-56.

The direct observation period was from October 2010 to March 2011. The first video-observation was made in August 2011 and the second videoobservation was performed one month later. The video-observations were used to test the intra-observer reliability. The intra-observer reliability was presented as percent agreement of each exposure interaction, in total fourteen interactions each for the neck and shoulder/arm.

\section{Results}

The mean observation time spent on assessing each worker was 45 minutes (range 30-120), waiting time and time for complementary questions included. When reassessing the work tasks using the video recordings the mean exposure observation time was 7 (range 2-10) minutes in video assessment one and 4 (range 2-5) minutes in video assessment two. No waiting time or complementary questions was included when reassessing.

The exposure scores varied between the different occupational groups as well as between workers within the occupational groups. The cleaners had the highest mean score in the neck and the workers at the hospital sterilization department had the highest mean score in the shoulder/arm (Table 1).

Table 1 Mean (range) exposure score in the neck and shoulder/arm in the different occupational groups.

\begin{tabular}{|c|c|c|}
\hline $\begin{array}{l}\text { Body-region/ } \\
\text { Occupational } \\
\text { group }\end{array}$ & Neck & Shoulder/arm \\
\hline Nurses & $13(8-16)$ & $29(26-34)$ \\
\hline Secretaries & $14(10-18)$ & $29(24-30)$ \\
\hline Sterilization & $15(12-16)$ & $43(36-52)$ \\
\hline Cleaners & $17(16-18)$ & $36(34-38)$ \\
\hline BMA & $14(8-16)$ & $30(18-44)$ \\
\hline
\end{tabular}

The percentage of time spent on the most common work task was in mean $62 \%$ (range 25-100), based on the workers self-reports.

Of the 23 workers observed, 18 rated their most common work task as also being the most strenuous for the neck/shoulder region. For the remaining five workers, the mean exposure score and ranges for the neck were higher in the most common work task compared to the most strenuous, 15 (range 12-16) versus 12 (range 8-14) (Figure 1), and the same pattern was found for the shoulder/arm, 32 (range 2638 ) versus 25 (range 20-32). 


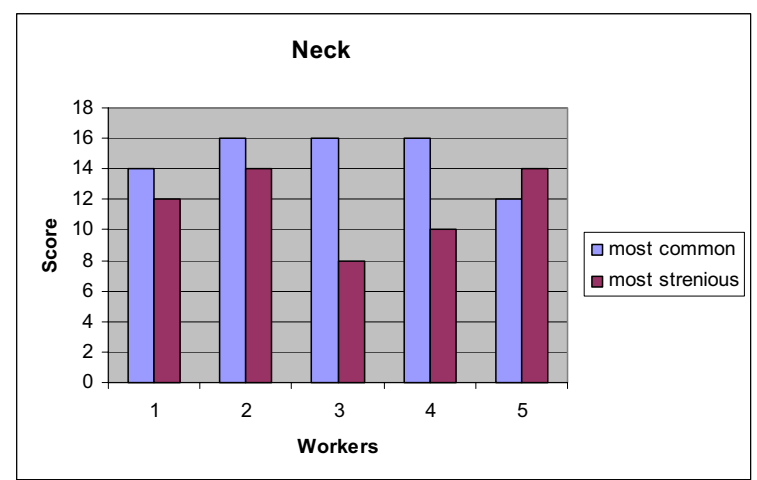

Figure 1 Differences in exposure scores between the most common versus the most strenuous work task in the neck between the five workers.

When testing intra-observer reliability between the two video assessments, 12 out of $14(86 \%)$ exposure interactions in the neck and 10 out of 14 (71\%) exposure interactions in the shoulder /arm showed an agreement.

\section{Discussion}

The QEC has been developed to be a quick and easy exposure assessment tool in ergonomics. In our study the mean observation time using QEC was 45 minutes, waiting time and time for complementary questions included. When making the same exposure assessment using the video recordings, the mean observation time were much shorter, 7 minutes in video assessment one and 4 minutes in video assessment two. However, in the observation time of the video recordings no waiting time or complementary questions was included. The longer direct observation time also can be explained by the waiting time when observing the operating-room nurses, because of their special working environment.

We found a variation in exposure score between workers in the same job as well as between different jobs. This variation supports that the QEC is sensitive enough to capture differences in exposure both between occupations as well as between workers within the same job.

The mean exposure score were higher both for the neck and shoulder/arm in the most common work task compared to the most strenuous work task for the neck/shoulder region. Duration is one risk factor involved in two out of two interactions when summa- rizing the total score for the neck, and in three out of five interactions for the shoulder/arm. This makes duration an important factor for the total summarized score in QEC. The most common work task is performed over longer time compared with the most strenuous work task, thus the total score becomes higher when summarized.

In our study QEC have a high percent of agreement in exposure interactions. This result support that the intra-observer reliability is good.

The reference guide was easy to embrace with clear background information and guidelines how to use QEC. When observing the different body regions of the worker in different work tasks, the instructions when using QEC are to select the worst case situation. This could lead to overestimation of the exposure. Another way could be to score the most common posture during the observation period.

We conclude that QEC is a quick and practical ergonomic exposure assessment tool that covers physical risk factors in body regions of the upper extremities with the greatest risk for work related musculoskeletal disorders.

\section{References}

[1] Bernard, B.P. (Ed.). Musculoskeletal disoprders and workplace factors. A critical review of epidemiologic evidence for work-related musculoskeletal disorders of the neck, upper extremity, and low back. National Institute for Occupational Safety and Health (NIOSH), Cincinnati, OH, 1997.

[2] da Costa BR, Vieira ER. Risk factors for work-related musculoskeletal disorders: A systematic review of recent longitudinal studies. Am J Ind Med 2010;53(3):285-323.

[3] Takala EP, Pehkonen I, Forsman M, Hansson GA, Mathiassen SE, Neumann WP, Sjogaard G, Veiersted KB, Westgaard RH, Winkel J. Systematic evaluation of observational methods assessing biomechanical exposures at work. Scand J Work Environ Health 2010 Jan; 36(1):3-24.

[4] Wells R, Mathiassen SE, Medbo L, Winkel J. Time - A key issue for musculoskeletal health and manufacturing. Appl Ergon 2007;38(6):733-44.

[5] David GC. Ergonomic methods for assessing exposure to risk factors for work-related musculoskeletal disorders. Occup Med (Lond) 2005;55(3):190-9.

[6] Li G, Buckle P. Current techniques for assessing physical exposure to work-related musculoskeletal risks, with emphasis on posture-based methods. Ergonomics 1999;42(5):674-95.

[7] David G, Woods V, Li G, Buckle P. The development of the Quick Exposure Check (QEC) for assessing exposure to risk factors for work-related musculoskeletal disorders. Appl Ergon 2008;39(1):57-69. 